\title{
BMJ open Observational study on factors related to health-promoting community activity development in primary care (frAC Project): a study protocol
}

To cite: March S, Ripoll J, Ruiz-Giménez JL, et al. Observational study on factors related to health-promoting community activity development in primary care (frAC Project): a study protocol. BMJ Open 2012;2:e001287. doi:10. 1136/bmjopen-2012-001287

- Prepublication history for this paper is available online. To view this file please visit the journal online (http://dx. doi.org/10.1136/ bmjopen-2012-001287).

Received 10 April 2012 Accepted 13 April 2012

This final article is available for use under the terms of the Creative Commons Attribution Non-Commercial 2.0 Licence; see http://bmjopen.bmj.com

For numbered affiliations see end of article.

Correspondence to Sebastià March; smarch@ ibsalut.caib.es

\section{ABSTRACT}

Introduction: According to Spanish health regulations, primary care professionals have the responsibility to carry out health-promoting community activities (CAs). However, in practice, their implementation is not as widespread as it should be. The aims of this study were to identify factors within the team, the community and the professionals that influence the development of these activities and to describe the community interventions in progress.

Methods and analysis: This study is an observational analytical retrospective study. The information will be collected from five Spanish regions: Catalonia, Madrid, the Balearic Islands, Navarra and Aragón. The authors will contact primary care teams (PCTs) and identify the CAs from the previous year. The research team will conduct a peer review whether the inclusion criteria are met. In the health centres where CAs are implemented, the authors will select professionals carrying them out and randomly select an identical number of professionals not doing these activities. In the centres where no CA is implemented, three professionals will be randomly selected. The selected professionals will complete the questionnaires for individual-level variables. Information about the registered population and the PCTs will be collected through questionnaires and secondary sources.

Outcomes: Variables will be collected from the community, the PCTs, the individual professionals and CAs.

Analysis: A descriptive analysis of all the variables will be carried out, along with a bivariate and a logistic regression analysis, with CAs being the primary outcome.

Ethics and dissemination: This study has been approved by the Research Ethics Committee of the

\section{ARTICLE SUMMARY}

\section{Article focus}

- There is much variability in the implementation of interventions to promote health at a community level in the Spanish primary healthcare.

- The study aims to establish which factors are related to such implementation, distinguishing between variables at the community and the primary care team level as well as professional factors at an individual level.

- There is scant research describing what is being done on health promotion CAs in a country with a highly developed primary healthcare.

\section{Key messages}

- A better understanding of those factors related to the implementation of these interventions could help health policymakers better understand the context of their uneven implementation and promote them.

- This study will lead to evaluate how the factors related to professionals, community and primary healthcare teams independently influence on the implementation of health promotion CAs.

Strengths and limitations of this study

- The study includes an operational definition of health-promoting CA resulting from a consensus of experts.

- Variables will be collected from the community, the primary care team and the professionals at an individual level from five Spanish regions.

- The main limitations of this study are related to the observational nature of its design and the difficulty to collect primary information of some measurements. 
Jordi Gol y Gurina Foundation in Barcelona and area 11 in Madrid. The questionnaire distributed to the professionals will be anonymous.

\section{INTRODUCTION}

Community action has been considered a key element in tackling the health-disease process and health promotion among populations since the International Conference at Alma-Ata in $1978 .{ }^{1}$ Its importance has been further emphasised in several international conferences. $^{23}$

Spanish healthcare reform in the 1980s gave Primary Health Care (PHC) the task of implementing healthpromoting community activities (CAs) through multidisciplinary teamwork throughout the country, which are accessible for the population. ${ }^{4}$ In fact, the specialty of the general practitioner, set up in Spain in 1978, is called Family and Community Medicine. ${ }^{5}$ This responsibility has been subsequently reasserted in healthcare legislation. ${ }^{6}$

The guidance for health promotion in primary care reflected in the law has not been sufficiently put into practice. ${ }^{7-9}$ Most of the PHC professionals have adopted a model of care, based on individual care, focused on disease and with very little teamwork. ${ }^{10}$ Nowadays, the implementation of CAs in Spanish health centres is unevenly distributed ${ }^{11}$ and is often implemented solely due to the voluntarism of the professionals. ${ }^{12}$ Many reasons for this have been identified. ${ }^{13-16}$

There is a lack of knowledge regarding the characteristics of the CAs currently implemented in Spain and the factors related to the involvement of PHC professionals in them. Literature on these factors is scant, although some research into factors related to the practice of primary prevention or health education can provide guidance. In these studies, the importance of factors concerning the professional such as sex and age $\mathrm{e}^{17-19}$; occupational category and situation ${ }^{20}$; specific education $^{20}{ }^{21}$; lack of motivation ${ }^{20}$; practice model (biomedical-psychosocial) ${ }^{22}$; features of the primary care team and the community where they work, including rural/ urban location ${ }^{23}$; satisfaction with the team ${ }^{22}$ or autonomy to manage priorities, timetables and space availability in the health centre ${ }^{24}$ has been highlighted. In the studies on professionals, the importance of attitudes and beliefs such as confidence in the efficiency of preventive actions, ${ }^{21} 22{ }^{25}$ self-efficacy when carrying them out ${ }^{202125}$ or their discourse about community participation $^{24}$ seems to have greater weight than their background. ${ }^{19}$ The social awareness of professionals, as measured by their political position, has also been demonstrated to be related to their involvement in $\mathrm{CAs}^{26}$ The undefined role of the health professional, unsure of the limits of their responsibilities, has been recognised as an important organisational factor. ${ }^{21} 22$ The most important factor according to professionals in Spain is the lack of time due to the healthcare burden. ${ }^{162022}$ All these factors have been separately observed in descriptive and, occasionally, qualitative studies. ${ }^{21} 22$

The purposes of this project were to explore how professional, primary care team (PCT) and the registered community elements are related to the design and implementation of health-promoting CAs and to describe the characteristics of the CAs that are implemented in Spain. In order to do so, the following objectives are proposed:

1. To determine the factors in the assisted community and the PCT related to the participation of a PCT team in CAs.

2. To identify the individual factors influencing the implementation of CAs by the primary care professionals at the health centre carrying them out.

3. To describe the factors related to the professionals, the assisted community and the PCT influencing the implementation of CAs by a PHC professional and to evaluate the influence of each of these factors.

4. To describe the identified CAs.

\section{METHODS AND ANALYSIS \\ Design}

This study is an observational analytical retrospective study. To accomplish the first objective, the PCT will be the unit of analysis and the centres developing CAs will be compared with those not applying them. For the second and third objectives, the unit of analysis will be the professionals. For the second objective, the professionals carrying out CAs will be compared with those who do not develop the activities in the same centres. For the third objective, the same professionals who develop CAs will be compared with the professionals who work in the centres not carrying them out. For the fourth objective, the study population will be the CAs identified in the health centres included in the study. Information regarding the professionals and the primary care team/ assisted community will be collected from different sources.

\section{Setting}

The information will be collected from five Spanish regions: Balearic Islands, Catalonia, Aragón, Madrid and Navarra. Each region has autonomy in the organisation of its health service, although there are some essential common features such as universal coverage or the basic services provided by the professionals. Each region is divided into health areas, which are covered by a variable number of health centres and which may have differing priorities and organisational systems. In the Balearic Islands, the area of Mallorca will be completely covered with 43 health centres. In Madrid, 28 health centres from area 1 will be studied. In Catalonia, information from the 63 health centres in the Barcelona metropolitan area will be collected. In Aragón, the information will be gathered from 45 health centres, which correspond to two sectors, Zaragoza and Huesca. Finally, in 
Navarra, consisting of only one area with 54 health centres, 15 centres will be randomly selected.

\section{Definition of CA}

One of the difficulties of doing research into CA lies in its very definition as it can be understood in different ways. With this in mind, the research team, with the collaboration of a group of experts, previously created the following conceptual definition ${ }^{27}$ : those intervention and participation activities of which take place in groups showing common characteristics, needs or interests with the aim of promoting health, improving quality of life and social well-being, boosting the capacity of people and groups to solve their own problems and meeting demands or needs in accordance with the following inclusion criteria:

- The health centre professionals participate in CAs, regardless of whether they are the promoters or not.

- The activities have to be part of a programme. Thus, they are not independent and isolated actions.

- The community actively participates in their design, implementation and/or assessment.

The following are excluded:

- Actions addressed only to diagnose specific health problems (screening programmes, check-ups, etc) when the community is just a passive receiver.

- Actions addressed to the prevention of a specific health problem through the application of a specific therapy (such as vaccinations) when there is no active participation of a sector of the community.

- Actions addressed only to the detection of health risk factors and addictions.

- Specific individual visits to the healthcare provider, even though they are related to health promotion (family planning, giving up smoking, etc), except in the case of being part of a broader project with the participation of a sector of the community.
- Actions addressed to groups, without contemplating the participation of a sector of the community in their organisation, which are limited to the transfer of information such as talks or advice about healthy habits.

From this conceptual definition, an operative one was developed to be used in this study. This definition includes the conditions a health promotion intervention needs to have to be considered a CA in an algorithm (figure 1). Therefore, it has to be a non-isolated activity, carried out in the last year, in which the professional has participated on behalf of the primary care team and in which the population shows a higher level of participation than the passive public or it is a cross-sector activity in which sectors other than health are collaborating.

Inclusion criteria are addressed to the PCTs that have been in operation for at least 1 year and for the professionals who have been working in the centre for at least 6 months and who are doctors, nurses, paediatricians or social workers.

\section{Subject selection}

The selection will take place in two phases (figure 2): a first one preselecting the centres and confirming the realisation of CAs and a second one selecting the professionals from each health centre.

\section{Preselection phase}

In each health area studied, the nursing managers from all health centres will be contacted and administered a short telephone questionnaire $(\mathrm{Q} 1)$ in which they will be asked about the participation of their PCT in CAs in the previous year. In the case of not being able to contact the nursing managers or an absence of CAs, the same questionnaire will be sent to the medical coordinator of the centre to verify the information. If one of them answers that they do indeed participate in CAs, they will
Figure 1 Algorithm of community activity (CA) confirmation.

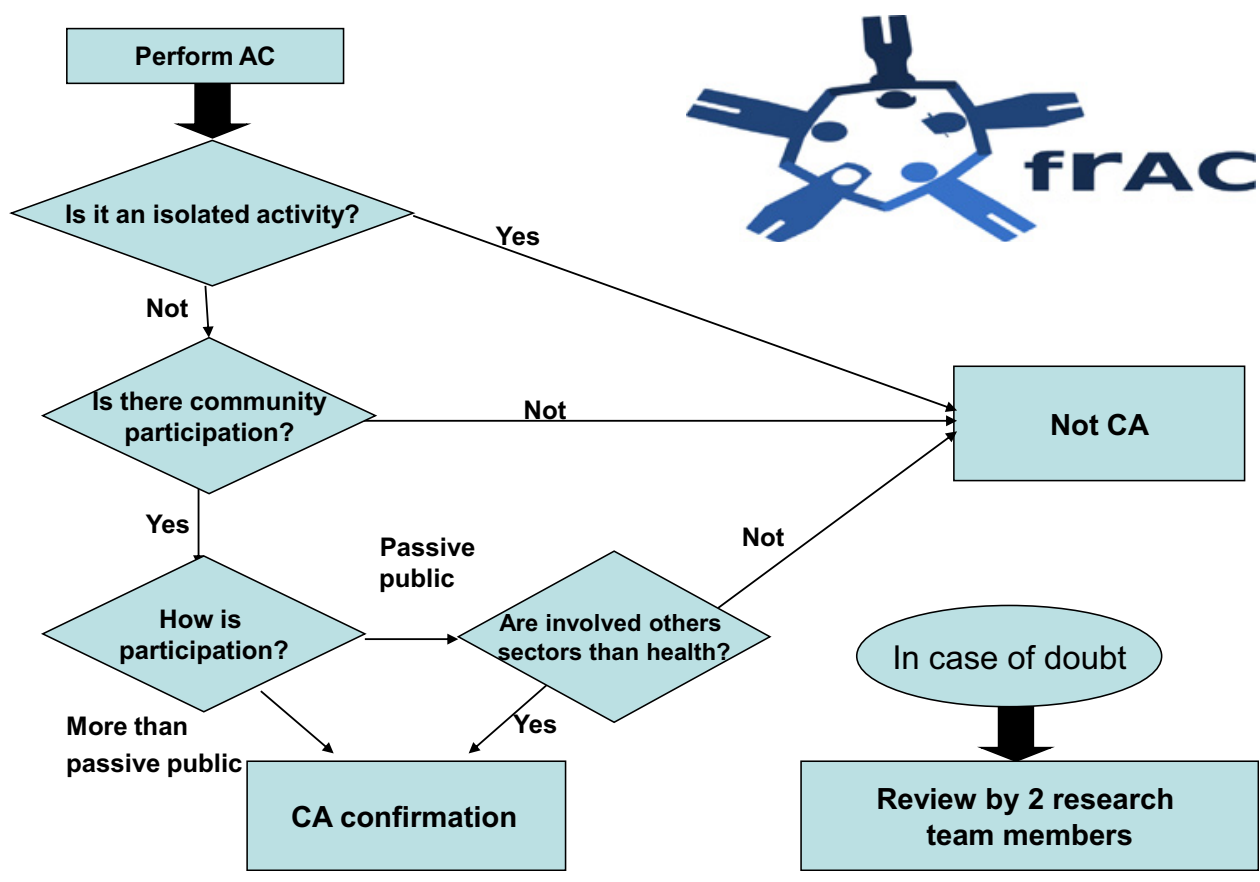


Figure 2 Algorithm for selection of cases and controls.CA, community activity; $\mathrm{PCT}$, primary care team.

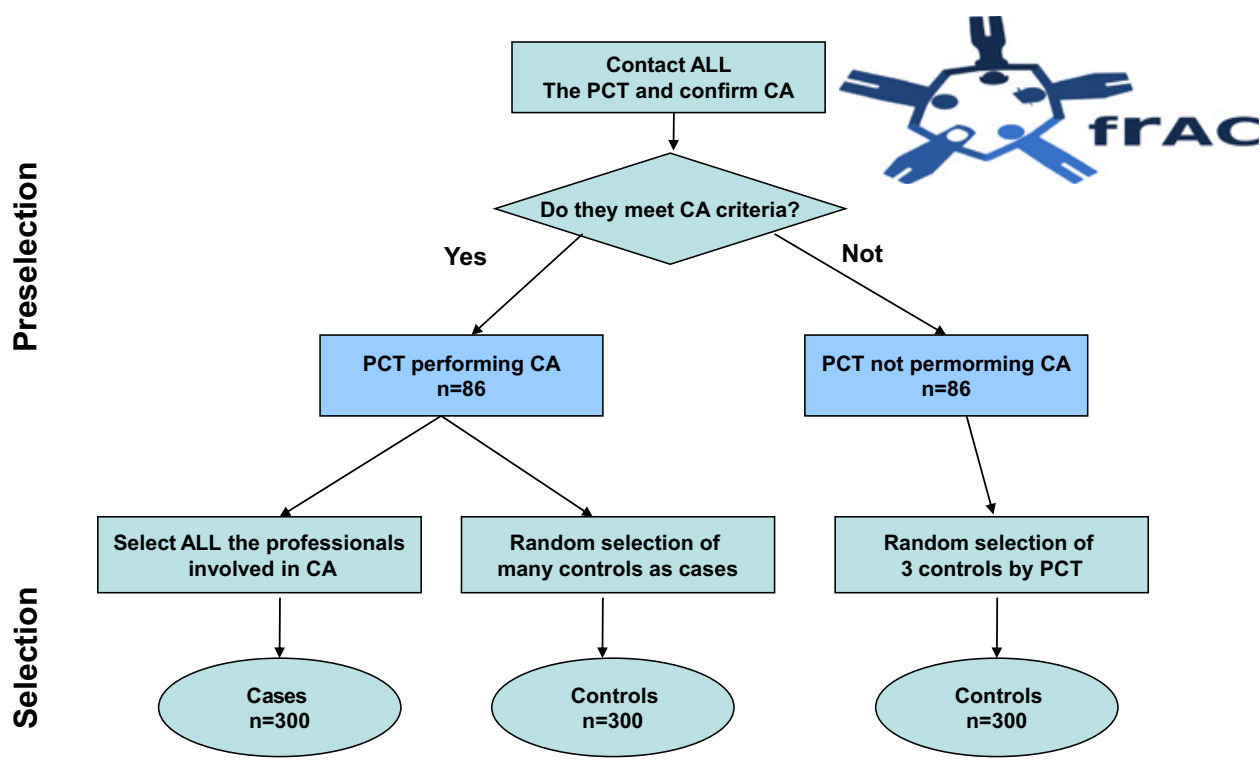

be asked to give a contact person for each CA. Moreover, some further questions detailed below regarding the health team and the assisted community will be asked.

Subsequently, a contact person for each CA will be identified and another questionnaire (Q2) will be given to them, which includes some questions at the beginning to confirm that the CA complies with the algorithm. Both questionnaires will be administered by trained staff and will include the definition of $\mathrm{CA}$ to facilitate understanding of the concept. However, in case of doubt, Q2 will be completed and qualitative information about CAs will be collected. Members of the research team will peer-review all this information. They will be responsible for the final confirmation of CA.

This questionnaire $\left(Q^{2}\right)$ will also collect information about the CA and the professionals involved. For each centre with confirmation of $\mathrm{CA}$, one centre with confirmation of absence of CAs will be chosen from the same area as the first one.

\section{Selection phase}

Once the realisation of $\mathrm{CA}$ is confirmed by a centre, the selection of professionals will take place. In the centres where CAs are developed, all CA participants will be selected with a maximum of 10 people per PCT. If there are more than 10, the selection will be done at random. In the same centres, an identical number of professionals not participating in CAs will be randomly selected. In the centres where no CAs are implemented, a minimum of three professionals will be selected at random. All the random selections will be done using a simple random sampling procedure with a computer program.

If a professional cannot be reached or he/she declines to answer, data regarding age, sex, occupational category and the reason for non-participation will be collected, and a substitute will be chosen by following the same randomisation procedure.

\section{Sample size calculation}

Due to the scarcity of information on the distribution of variables under study, a 50\% proportion on the PCT, which develop CA, is supposed related to the low-middle socioeconomic community variable. Sample calculation was based on estimated results of the first objective; with an OR of 2.5 and a relative precision of $50 \%, 72$ PCTs will be needed in each group.

For objectives two and three, we hypothesised that $50 \%$ of professionals doing CAs are supposed to have specific training on the realisation of CAs. For an OR of 2 with $40 \%$ precision, it will be necessary to interview 126 professionals per group. Assuming a multilevel analysis for objective 3 , a design effect of 2 is applied so it will be necessary to interview 252 professionals per group. Finally, $20 \%$ is added to the calculated sample to mitigate possible losses due to lack of data. Thus, it will be necessary to interview 900 professionals (300 per group) from 172 PCT (86 per group).

\section{Information collection}

The information will be gathered from three different questionnaires (table 1). The selected professionals will be informed of their selection either by the nursing manager or directly by a member of the research team. A self-administered questionnaire (Q3) will be handed to them. A variety of strategies for sending the questionnaires to the recipients will be used. Where possible, the professionals will be called to a project explanatory meeting where the questionnaires will be handed to them. In more distant centres, the questionnaires will be sent by internal mail with a double envelope.

At the same time, information from secondary sources about the health centre and the assisted population characteristics will be collected from the regional health system's databases. 
Table 1 Sources of information used in the study

\begin{tabular}{lll}
\hline & Respondent & Level of information \\
\hline Questionnaire 1 (Q1) & Health centre coordinator and/or nurse manager & PCT and assisted community \\
Questionnaire 2 (Q2) & Community activity contact person & Community activity \\
Questionnaire 3 (Q3) & Health professional & Individual professional \\
Data from secondary sources & - & PCT and assisted community \\
\hline PCT, primary care team. & &
\end{tabular}

\section{Collected outcomes}

The dependent outcome for the three main objectives is the realisation or otherwise of CAs according to the above-mentioned definition criteria. The independent outcomes are as follows:

\section{Assisted community}

Through an interview with the centre coordinator or the nursing manager $(\mathrm{Q} 1)$, or through the health regional systems databases, the following outcomes will be collected: percentage of women, percentage of people older than 65 years, percentage of children younger than 16 years, per cent from outside the European community and the size of the town they live in. The health centre managers will assess the area (urban/ rural) where the centre is located, the general socioeconomic level of the population (high, medium-high, medium, medium-low, low) and their level of collaborative partnerships (high, medium and low). Geographical dispersion will be assessed with an ordinal index used by the national health system for resource allocation (G1 more compact population and G4 more dispersed). They will also be asked about the existence of health councils, which are structures of community participation in health outlined in the general health $\mathrm{law}^{4}$ and in the consecutive regional adaptations, with various levels of development in the different regions of the country. ${ }^{28}$

\section{Primary healthcare team}

Information on the size and composition of the professional team will be collected from secondary sources to calculate the percentage variables of nurse, doctor and paediatrician attendance burden. The size of the health centre's registered quota will be collected and patientprofessional, doctor-patient, nurse-patient and doctor-nurse ratios will be calculated. The opening year of the health centre will be noted. They will be asked, through Q1, if they have room in the centre to carry out group activities, whether it is an accredited teaching centre (with the following options: doctors, undergraduate nurses and postgraduate nurses), whether they have a person in the PCT responsible for health education or similar and, in the case that it exists, whether the PCT collaborates with the area health council. The coordinator of the centre will be asked to assess the professional relationships between doctors and nurses, doctors and social workers, and nurses and social workers on a scale from 1 to 5 ( 1 , bad relationship; 5 , excellent relationship).

\section{Professionals}

In the questionnaire for the professionals (Q3), information on sex, age, profession and working situation (steady job, temporary worker and other) will be collected with some other data on their attendance burden: size of assigned quota, average of daily office hours and house visits and average of time per visit in the centre and homes. The following information will be also collected: whether they act as tutors (of medicine or nursing), whether they have collaborated on any research project in the previous 5 years, whether they have received any specific training on the realisation of CAs in the same period, their year of graduation, the year when they started work in primary care and the year when they started to work in the health centre where they are now.

Regarding work organisation and satisfaction, they will be asked if they have enough autonomy to manage their timetables and priorities, the possibility of having a colleague to cover their office hours while they engage in CAs and whether they can count on other professionals within their PCT to support them in the realisation of CAs. They will be asked to assess the working environment, their work satisfaction and the space available to carry out group activities on a $1-5$ scale ( 1 , low; 5 , high). They will also be asked to assess, on a scale of $1-5$, their self-perceived capacity to implement CAs.

The literature review indicated the importance of opinions and attitudes, ${ }^{19-25}$ and they will be investigated by formulating some statements in which the professionals will indicate their degree of agreement on a Likert scale of four options: from strong disagreement to strong agreement. The statements are related to the following topics: confidence in the efficacy of CAs, motivation to develop CAs, citizen participation in decision-making about health, the need to boost citizen participation in health, the responsibility of PCT in community health work, the definition of the professional's role, the connection between health education and community participation and the efficacy of health councils in boosting CA. Moreover, a scale will be administered to identify the style of practice of the professional in PHC (biomedical-psychosocial), ${ }^{29}{ }^{30}$ which consists of four items about their attitude regarding the demands of psychosocial and clinical assistance at work.

In order to complete the information about attitudes, a variable called social awareness was created, which will be collected through the following proxy variables: participation during the last year in activities in the neighbourhood where he/she works, involvement in any 
non-governmental organisation or civil association and political views. For the final one, a visual scale adapted from the ones used in the Spanish Center of Sociological Research in their monthly barometers will be used. The scale has a 0 in the centre and 5 in the left and right extremes, and the surveyed person is asked to locate their political orientation on the left-right axis.

\section{Community activities}

The descriptive information about CAs will be collected through a questionnaire $(\mathrm{Q} 2)$, which will be completed by the person in charge of each CA. The questions will be related to the problem the CA is covering; the target population; the number of professionals from the PCT involved; for how long and the place where it is being carried out (multiple choice: neighbourhood premises, street, health centre, educative centres, NGO premises, other); whether there is an existing assessment; whether any specific theoretical perspective is being used and, if so, which one; and whether the CA is registered with any health promotion network among those already existing in Spain. The nature of community participation will also be checked: whether they do it as public-receiver, as consultant-claimant or as a planner. In the final option, it will be determined whether the participation has taken place in the design, in the process and/or in the CA assessment. Moreover, they will be asked about the collaboration of other health and non-health sectors such as hospitals, education, social services, NGOs, civil associations and administration (local, regional or state). The person responsible for each CA will be asked to assess, from 1 to 5 , the degree of involvement ( 1 means less involvement; 5 means greater involvement) of the administration, the community and the other PCT professionals in the CA.

\section{Statistical analysis and data management}

The questionnaires will be processed with the automated data reading software Teleform. All information from the questionnaires and record sheets will be entered in an Access database. Filters and rules of validation will be used to avoid errors and to ensure good quality data. The information will be centrally monitored by the Balearic Islands team, which will correct any errors and add missing values from the databases by contacting the rest of the regions.

The analysis procedure will be similar for the three main objectives. A descriptive analysis of all considered variables will be carried out. The distribution of the variables in each of the compared groups, expressed as percentages and averages with $95 \%$ CIs (medians and P25-P75 when the continuous variables do not follow a normal distribution), will be contrasted, and the relationship between the independent variables with the realisation of CAs will be assessed with the $\chi^{2}$ test for categorical variables and with the Student $t$ test for continuous variables if they adjust to a normal distribution or with the Mann-Whitney $U$ test if they do not.
The strength of association will be expressed by using the crude ORs and with $95 \%$ CIs.

A multivariate analysis will be performed using the logistic regression technique, in which the dependent variable will be the realisation or otherwise of CAs. For the construction of the model, the starting point will be the saturated model, introducing all those variables with a significance $p<0.20$ in the bivariate analysis and those which are considered to influence the model. Variations in the model will be tested, and those variables not having a significance $p<0.05$ in the Wald test will be eliminated when that does not involve a remarkable variation in the standardised $\beta$ coefficients of the variables in the model. New models will be compared with previous models by using a method of maximum likelihood until the most suitable explicative model is selected.

To accomplish the third objective, the need to try a multilevel analysis will be considered, where the first level would be the professionals and the second would be the PCT. The hierarchical models will be compared with the non-hierarchical ones to see which one adjusts better, and in the case that the first one is better, the weight of the factors in each level will be determined.

To achieve the fourth objective, a descriptive analysis of the CA characteristics as a whole and divided into regions will be carried out.

The statistical analysis will be performed with the support of the Epidat 3.1 and SPSS V.18. programs.

\section{Limitations}

The retrospective analytical design allows the exploration of many variables simultaneously, although it hampers the possibility of determining causal relationships. This could be considered a study limitation because, for some variables, a temporal sequence cannot easily be established. The CA definition has many meanings and interpretations, which can complicate result reliability. We have tried to control this difficulty by using a definition from a consensus of experts, which was included in an algorithm to facilitate inclusion. All doubtful cases were peer-reviewed by a member of the research team. Another limitation could be the common problems in recruiting professionals. Consequently, a plan to present the project to the management offices has been prepared, and there are research team members in each of the study participant regions.

Some variables are difficult to determine with the available information, such as the level of collaborative partnerships or the socioeconomic level of the assisted community. This has been dealt with by accepting the answers of the health centre managers about these topics as valid, considering that they are sufficiently close to the population under their care.

\section{Ethics and dissemination}

The study has been approved by the research commissions of all areas where it is going to be carried out as well as by the Research Ethics Committee of the Jordi Gol y Gurina Foundation in Barcelona and area 11 in Madrid. 
The study was also presented to all management offices of the primary care participants before being presented to the professionals and received their approval.

The questionnaire distributed to the professionals, which contained confidential variables, will be anonymous, and both the professionals and the health centre will be encoded so that the researchers cannot know its origin.

\section{Relevance and dissemination}

The research team's hypothesis is that the factors related to the professional have greater influence than those of the PCT and the assisted community; especially those factors related to health promotion opinions and beliefs. This would suggest that primary care professionals in Spain, regardless of the legislation defining their responsibilities, adapt their practice to their individual beliefs, overlooking the idea of multidisciplinary teamwork, which forms the basis of primary care.

Moreover, the results of this study will provide information on the health promotion interventions that primary healthcare centres are performing at the community level in Spain.

There is scant research on this topic. Further study can help to boost health-promoting community interventions and primary care itself and also to discover more about what leads the professionals to develop specific practices and orientations.

\section{Author affiliations}

${ }^{1}$ Primary Care Research Unit of Mallorca, Baleares Health Services-IbSalut, Palma de Mallorca, Spain

${ }^{2}$ Vicente Soldevilla Primary Health Center, Madrid Health Services, Madrid, Spain

${ }^{3}$ El Carmel Primary Health Center, Catalonian Health Services Cat-Salut, Barcelona, Spain

${ }^{4}$ Oliver Primary Health Center, Aragon Health Services, Zaragoza, Spain

${ }^{5}$ Navarra Public Health Institute, and Public Health and Epidemiology CIBER, Pamplona, Spain

${ }^{6}$ School of Nursing and Physiotherapy, Balearic Islands University, Palma de Mallorca, Spain

${ }^{7}$ Son Gotleu Primary Health Center, Baleares Health Services-IbSalut, Palma de Mallorca, Spain

${ }^{8}$ San Agustí Primary Health Center, Baleares Health Services-IbSalut, Palma de Mallorca, Spain

${ }^{9}$ Loeches Primary Health Center, Madrid Health Services, Madrid, Spain

${ }^{10}$ Estrecho de Correa Primary Health Center, Madrid Health Services, Madrid, Spain

${ }^{11}$ DA Sureste Primary Health Center, Madrid Health Services, Madrid, Spain

${ }^{12}$ Freelance, Madrid, Spain

${ }^{13}$ Madrid Society of Family Medicine and Community (SOMAMFYC), Madrid, Spain

${ }^{14}$ Sant Rafel Primary Health Center, Catalonian Health Services Cat-Salut, Barcelona, Spain

${ }^{15}$ Primary Care Health Research Institute IDIAP Jordi Gol, Barcelona, Spain

${ }^{16}$ Delicias del Sur Primary Health Center, Aragon Health Services, Zaragoza, Spain

${ }^{17}$ Primary Care Research Unit, Aragon Health Services, Aragon, Spain

${ }^{18}$ Zaragoza Public Health Provincial Subdirection, Zaragoza, Spain

${ }^{19}$ Arrabal Primary Health Center, Aragon Health Services, Zaragoza, Spain

${ }^{20}$ Public Health Department, Balearic Islands Health Department, Palma de Mallorca, Spain

Acknowledgements We thank Joan Llobera and Magdalena Esteva for their contributions and critical reviews of the manuscript.
Contributors SM and JR led the design and developed of the study. MR has the original idea. All the signing authors participated in the design and development of the study, have read the manuscript critically, made contributions and approved the final version. SM and JR will perform the statistical analysis. SM, JLR-G, IMG, CBBA and LES will coordinate the development of the study in their regions.

Funding This study is supported by a grant from the Carlos III Health Institute (PI07/90383; PI07/90925; PI07/90636). It has also received the support of the Health Promotion and Preventive Activities in Primary Health Care Research Network (IAPP network), supported by the Ministry of Health and Consumer Affairs (ISCIII RD 06/0018/0036) as well as by Interuniversity Institute of Sciences of Health Research (IUNICS). Also, SM is contracted with the aid of a grant to stabilise employment in heath research from the Carlos III Health Institute and was benefited from a grant for further studies from the same institution.

\section{Competing interests None.}

Ethics approval Ethics approval was provided by Fundación Jordi Gol y Gurina from Barcelona and health area 11 from Madrid.

Provenance and peer review Not commissioned; internally peer reviewed.

\section{REFERENCES}

1. World Health Organisation. Declaration of Alma Ata. WHO, 1978. http://www.who.int/hpr/NPH/docs/declaration_almaata.pdf

2. World Health Organisation. The Ottawa charter for health promotion. Ottawa: World Health Organisation,1986.

3. World Health Organisation. Declarations of Jacarta. WHO, 1997. http://www.who.int/hpr/NPH/docs/jakarta_declaration_sp.pdf

4. Ley General de Sanidad 14/1986, de 25 de abril. Boletín Oficial del Estado, $n^{\circ} 102$ (29/4/1986).

5. Real Decreto $3303 / 1978$, de 29 de diciembre de regulación de la Medicina de Familia y Comunitaria como especialidad de la profesión médica. Boletín Oficial del Estado n²9 (2/2/1979).

6. Real Decreto $1030 / 2006$ del 15 de septiembre por el que se establece la cartera de servicios comunes del Sistema Nacional de Salud y el procedimiento para su actualización. Boletín Oficial del Estado, n²22, (16/9/2006).

7. Irigoyen J. La participación comunitaria en salud: Una reformulación necesaria. In: Aranda JM, ed. Nuevas perspectivas en Atención Primaria de Salud. Madrid: Diaz de Santos 1994:183-221.

8. Gérvas J, Pérez-Fernández M, Palomo-Cobos L, et al. Veinte años de reforma de la Atención Primaria en España. Valoración para un aprendizaje por acierto/error. Madrid: Ministerio Sanidad y Consumo, 2005. http://www.msc.es

9. Regato P, Domínguez J, Sancho M. Coordinación sanitaria y abordaje comunitario en atención primaria. In: Navarro V, Martín-Zurro A, eds. La Atención Primaria de Salud en España y en sus comunidades autónomas. Barcelona: Semfyc, 2009:149-68.

10. Turabián JL, Pérez B. Grandes misterios. ¿Puedes ver al monstruo del lago Ness?: el modelo biopsicosocial y las actividades comunitarias. Aten Primaria 2007;39:261-4.

11. Soler Torroja M. Promoción de la salud, atención comunitaria y medicina de familia. Aten Primaria 2005;36:355-7.

12. Astray L. La intervención comunitaria en la encrucijada (editorial). Aten Primaria 2003;32:447-50.

13. Irigoyen J. Perspectivas de la participación en salud después de la reforma gerencialista. Comunidad 2004;7:73-9.

14. Gutierrez M, Azpeitia ME, Vergara I. Actuar con la comunidad... ¿Una asignatura pendiente en Osakidetza? Comunidad 2004;7:63-7.

15. Ruiz-Giménez Aguilar JL, Domínguez Bidagor J. Reflexiones sobre «lo comunitario» en nuestro sistema sanitario. Aten Primaria 2006;38:421-4.

16. Guldan GS. Obstacles to community health promotion. Soc Sci Med 1996;43:689-95.

17. Maheux B, Béland F. Changes in students sociopolitical attitudes during medical school: socialization or maturation effect? Soc Sci Med 1987;24:619-24.

18. Maheux B, Dufort F, Lambert J, et al. The professional attitudes and clinical practices of men and women generalists. Can Fam Physician 1989;35:59-63.

19. Maheux B, Pineault R, Lambert J. Factors influencing physicians' preventive practices. Am J Prev Med 1989;5:201-6.

20. Diaz G. Factores relacionados con la práctica de educación sanitaria por los médicos de Atención primaria de Galicia. Rev Esp Salud Publica 2001;75:529-40. 
21. Myrand A, Beehler G, Kuo C, et al. Explaining the de-prioritization of primary prevention: physicians' perceptions of their role in the delivery of primary care. BMC Public Health 2003;3:5.

22. Duro Martínez JC. Actitudes de los profesionales de atención primaria de Madrid sobre los grupos (In Spanish). Aten Primaria 2005;36:71-7.

23. Sáez S, Marqués $\mathrm{F}$, Pallarés $\mathrm{J}$, et al. Perfil de los profesionales de la salud de Lleida respecto a la educación para la salud (EpS) (In Spanish). Aten Primaria 1999;24:157-61.

24. Pérez-Franco B, Turabián JL, Magaña E, et al. Factores asociados a la participación comunitaria en la atención primaria de la provincia de Toledo. Aten primaria 1993;12:339-44.

25. Cheng TL, Hewitt TG, Savageau JA, et al. Determinants of counseling in primary care pediatric practice. Arch Pediatr adolesc 1999;153:629-35.
26. Maheux $B$, Pineault $R$, Béland $F$, et al. Factors influencing physicians' orientation toward prevention. Am J Prev Med 1987;3:12-18.

27. March S, Ramos M, Soler M, et al. Revisión documental de experiencias de actividad comunitaria en atención primaria de salud. Aten Primaria 2011;43:289-96.

28. Aguilar Ibáñez MJ. La participación comunitaria en salud: Mito o realidad? Madrid: Díaz de Santos, 2001.

29. Martínez-Cañabate T. Modelo multinivel explicativo de la utilización de las consultas de atención primaria en Andalucia. PhD thesis. Granada University, 2007. http://hera.ugr.es/tesisugr/16905234.pdf

30. Mira JJ, Llinás G, Gil V, et al. Validación de un instrumento para identificar estilos de práctica profesional del médico de atención primaria. Aten Primaria 1998;21:14-22. 